\title{
Una relación de homogeneidad entre términos heterogéneos. El concepto de homogeneidad en el capítulo del esquematismo de la Crítica de la razón pura
}

\author{
MARTÍN ARIAS AlBisu \\ CONICET (Consejo Nacional de Investigaciones Científicas y Técnicas) \\ arias.martin@gmail.com
}

Resumen: El presente artículo se ocupa del concepto de homogeneidad (Gleichartigkeit) empleado por Kant en el capítulo del esquematismo de la Crítica de la razón pura. Se discuten dos artículos recientes dedicados al problema, y se procura demostrar que, según Kant, la homogeneidad consiste en una relación entre dos términos heterogéneos (la categoría y la multiplicidad empírica) conseguida mediante la introducción de un tercer elemento (el esquema trascendental). Sostenemos que esta peculiar relación de homogeneidad no implica una supresión de la heterogeneidad (en el sentido usual del término) entre los términos vinculados por ella.

Palabras clave: entendimiento, sensibilidad, categoría, intuición

\begin{abstract}
This paper deals with the concept of homogeneity (Gleichartigkeit) used by Kant in the schematism chapter of the Critique of Pure Reason. I discuss two recent papers that address the same problem. I show that, according to Kant, homogeneity consists in a relation between two heterogeneous terms (the category and the empirical manifold) made possible by the introduction of a third element (the transcendental schema). I claim that such a peculiar relation of homogeneity does not entail the suppression of the heterogeneity (in the usual meaning of the word) between the terms linked by it.

Key words: understanding, sensibility, category, intuition
\end{abstract}

Me propongo ofrecer una interpretación del concepto de homogeneidad (Gleichartigkeit) empleado por Kant en el capítulo "Del esquematismo de los conceptos puros del entendimiento"1 de la Crítica de la

${ }^{1}$ A 137-147/B 176-187. Las referencias a la Crítica de la razón pura son dadas, como es habitual, según la paginación de las ediciones originales. Con "A" hacemos referencia a la primera edición (1781), y con "B" a la segunda (1787). Tanto para la primera Crítica como para otras obras kantianas, me he servido de los textos incluidos en Kant's gesammelte Schriften, edición de la Königlich Preußischen Akademie der Wissenschaften, Berlín, 1902 y ss. Se hará referencia a esta edición de conjunto con la abreviatura "AA" (Akademie-Ausgabe) seguida del número de tomo (en romanos) y de página (en arábigos). La Crítica de la razón pura se cita siempre según la traducción de Mario Caimi; cuando se hace referencia a pasajes de la Lógica Jäsche se incluyen los números de página de la traducción al castellano de María Jesús Vázquez Lobeiras (véanse en la bibliografía las referencias completas).

Diánoia, volumen LIV, número 63 (noviembre 2009): pp. 71-88. 
razón pura. Esta investigación incluirá un análisis de los pasajes más relevantes y un examen de dos artículos recientes dedicados al problema. ${ }^{2}$ Concluirá que el concepto kantiano de homogeneidad no guarda relación con el significado habitual del término. ${ }^{3}$ En efecto, la homogeneidad kantiana consiste en una relación sostenida por dos términos heterogéneos mediante un tercer elemento. Dicho en otras palabras, entre la categoría y la multiplicidad empírica se establece una relación de homogeneidad en virtud de la introducción de un tercer término, a saber, el esquema trascendental. Lo peculiar de esta relación de homogeneidad radica en que los elementos vinculados mediante ella permanecen heterogéneos en el sentido usual del término.

Comenzaré con una breve exposición de la problemática abordada por la doctrina del esquematismo trascendental. De esta manera se introducirá el marco conceptual en el que adquiere sentido el concepto de homogeneidad y, a la vez, se mostrará el interés que presenta el análisis del concepto.

El capítulo del esquematismo se propone mostrar cómo es posible la aplicación de las categorías a los fenómenos. A decir verdad, Kant utiliza tanto el término "subsunción" (Subsumtion) como la expresión "aplicación" (Anwendung). Con "subsunción" se hace referencia a la identificación de un caso como caso particular de un concepto, mientras que con "aplicación" se designa la acción de partir del concepto y hallar el caso particular al cual debe aplicarse. Como puede apreciarse, se trata en realidad de uno y el mismo problema.

Kant señala que la heterogeneidad que se presenta entre las categorías y la multiplicidad empírica parece tornar impracticable la subsunción de la segunda bajo las primeras. ${ }^{4}$ Las categorías son conceptos que

${ }^{2}$ Los trabajos que principalmente tendré en cuenta son los siguientes: D. Lohmar, "Kants Schemata als Anwendungsbedingungen von Kategorien auf Anschauungen. Zum Begriff der Gleichartigkeit im Schematismuskapitel der Kritik der reinen Vernunft", y M. Caimi, "Der Teller, die Rundung, das Schema. Kant über den Begriff der Gleichartigkeit". Hasta donde sé, estos dos artículos son los únicos que se ocupan con detenimiento del problema de la homogeneidad. Las referencias completas se encuentran en la bibliografía incluida al final del presente artículo.

${ }^{3}$ El término alemán "Gleichartigkeit" es muy similar al término castellano "homogeneidad". Significa principalmente "semejanza pronunciada" y "pertenencia al mismo género o procedencia de éste". Me he servido de Der Digitale Grimm. Deutsches Wörterbuch von Jacob und Wilhelm Grimm y Wahrig Deutsches Wörterbuch.

${ }^{4}$ La lectura de los dos primeros párrafos del capítulo del esquematismo no permite identificar inequívocamente cuáles son los términos de la relación de subsunción. Es claro que los conceptos bajo los cuales debe subsumirse el factor empírico 
se originan íntegramente en el entendimiento y expresan la espontaneidad del mismo. Considerados aisladamente, estos conceptos no tienen siquiera vínculo alguno con las formas de la sensibilidad, a saber, las intuiciones puras del espacio y el tiempo. El contenido de las categorías -es decir, lo pensado en ellas- es, entonces, heterogéneo con respecto a las intuiciones sensibles. Por su parte, la multiplicidad empírica es acogida en la sensibilidad en virtud de la afección de ésta por parte de un ente independiente del sujeto. Considerada en sí misma, la multiplicidad empírica es un factor meramente dado y aún no determinado por la espontaneidad del sujeto.

Las categorías y la multiplicidad empírica son, entonces, heterogéneas. La subsunción de la segunda bajo las primeras no puede tener lugar inmediatamente, dado que ello sería "contrario a la lógica". ${ }^{5} \mathrm{Se}$ hace necesaria la intervención de elementos mediadores, a saber, los esquemas trascendentales. La función de un esquema trascendental consiste en producir homogeneidad entre una categoría y la multiplicidad empírica a fin de tornar posible la aplicación de la primera a la segunda. ${ }^{6}$

son las categorías; ahora bien, no es evidente cuál es exactamente el factor empírico. Kant menciona tanto las intuiciones empíricas y sensibles como los fenómenos y los objetos (cfr. A 137/B 176). Hay que señalar que la aplicación de las categorías es un momento constituyente de los objetos empíricos; por lo tanto, el factor empírico al que se aplican las categorías no puede ser sino la multiplicidad empírica dada en una intuición. Es probable que, en los textos mencionados, el concepto de fenómeno (Erscheinung) tenga el sentido que se asigna al término en A 20/B 34: "El objeto indeterminado de una intuición empírica se llama fenómeno." En esta acepción del término, "Erscheinung" equivale a multiplicidad empírica. En efecto, ambos términos hacen alusión a un factor empírico aún no determinado por las categorías. (Por otra parte, en A 248-249 se sugiere que el objeto indeterminado debería llamarse "Erscheinung", mientras que el objeto fenoménico constituido por las categorías debería ser denominado "Phänomenon". Como señala Paton, Kant se desentiende a menudo de esta distinción —cfr. H.J. Paton, Kant's Metaphysic of Experience, t. I, p. 96, n. 7-; en efecto, Kant denomina en varios pasajes "Erscheinung" a lo que según, el texto comentado, debería llamar "Phänomenon"; $c f r$., por ejemplo, A 246/B 303.) Por último, nótese que mediante la aplicación de las categorías a la multiplicidad empírica ésta se constituye como objeto empírico. El objeto empírico determinado es constituido en virtud de tal aplicación; por ende, sólo en un sentido amplio de "objeto" puede afirmarse que las categorías se aplican a objetos.

${ }^{5}$ Carta a J.H. Tieftrunk del 11 de diciembre de 1797; AA, XII, 224. La traducción es mía.

${ }^{6} \mathrm{El}$ lector puede encontrar presentaciones de la doctrina kantiana del esquematismo en numerosos comentarios. La bibliografía sobre esquematismo es hoy en día muy vasta. Se mencionarán algunas obras importantes por uno u otro motivo. 
La exposición precedente pone en evidencia la importancia del concepto de homogeneidad. La filosofía crítica establece una distinción entre dos facultades cognitivas fundamentales, a saber, entendimiento y sensibilidad. Estas facultades deben operar conjuntamente a fin de que haya conocimiento objetivo. ${ }^{7}$ Ahora bien, la colaboración entre ambas facultades requiere que se establezca un vínculo de homogeneidad entre representaciones que son inherentes a ellas (las categorías y la multiplicidad empírica dada en una intuición). Por lo tanto, un examen del concepto de homogeneidad puede aportar precisiones acerca del modo en que se relacionan las facultades cognitivas en cuestión.

A continuación se citan los pasajes más relevantes para la comprensión del concepto de homogeneidad en el marco del capítulo del esquematismo. ${ }^{8}$

(1) En todas las subsunciones de un objeto bajo un concepto, la representación del primero debe ser homogénea (gleichartig) con el último; es decir, el concepto debe contener aquello que está representado en el objeto que hay que subsumir bajo él; pues esto, precisamente, significa la expresión: un objeto está contenido bajo un concepto. (A 137/B 176)

Hasta donde sé, la única exposición sistemática del problema del esquematismo en el desarrollo filosófico de Kant es: R. Daval, La Métaphysique de Kant. Perspectives sur la métaphysique de Kant d'après la théorie du schématisme. Dos artículos destacables por su abordaje riguroso y documentado de la doctrina del esquematismo trascendental (esquematismo de las categorías) son: W. Detel, "Zur Funktion des Schematismuskapitels in Kants Kritik der reinen Vernunft", y K. Düsing, "Schema und Einbildungskraft in Kants Kritik der reinen Vernunft". Como es sabido, Kant presenta en el capítulo del esquematismo elementos para una teoría del esquematismo de los conceptos empíricos y matemáticos (cfr. A 140-142/B 179-181). Para el estudio del esquematismo de los conceptos empíricos son dignos de mención: L. Chipman, "Kant's Categories and their Schematism", y R.B. Pippin, "The Schematism and Empirical Concepts". Para el esquematismo de los conceptos matemáticos, véase: A. Ferrarin, "Construction and Mathematical Schematism. Kant on the Exhibition of a Concept in Intuition", A.T. Winterbourne, "Construction and the Role of Schematism in Kant's Philosophy of Mathematics", y M. Young, "Construction, Schematism, and Imagination". Por último, debo señalar que en este artículo no me propongo ofrecer una interpretación exhaustiva del concepto de esquema trascendental. Este concepto se determinará únicamente en la medida en que sea necesario para comprender el concepto de homogeneidad.

${ }^{7}$ Cfr. A 51-52/B 75-76.

${ }^{8}$ No se examinará aquí el principio de homogeneidad (Gleichartigkeit) introducido por Kant en su tratamiento del uso regulativo de las ideas de la razón ( $c f r$. A 657/B 685). El concepto de homogeneidad es empleado allí en un marco conceptual que no guarda relación con la doctrina del esquematismo trascendental. 
(2) Así, el concepto empírico de un plato tiene homogeneidad (Gleichartigkeit) con el [concepto] puro geométrico de un círculo, pues la redondez, que está pensada en el primero, se puede intuir en el último. (A 137/B 176)

(3) Ahora bien, conceptos puros del entendimiento son completamente heterogéneos en comparación con intuiciones empíricas (y en general, con [intuiciones] sensibles), y nunca pueden ser hallados en intuición alguna. Entonces, ¿cómo es posible la subsunción de las últimas bajo los primeros, y por tanto, la aplicación de la categoría a fenómenos, puesto que nadie dirá: ésta, $p . e j .$, la causalidad, puede ser intuida también por los sentidos y está contenida en el fenómeno? (A 137-138/B 176-177)

(4) Ahora bien, está claro que debe haber un tercero, que debe estar en homogeneidad (Gleichartigkeit), por una parte, con la categoría, y por otra parte, con el fenómeno, y que hace posible la aplicación de la primera al último. Esta representación mediadora debe ser pura (sin nada empírico), pero [debe ser], por una parte, intelectual, y por otra parte, sensible. Una [representación] tal es el esquema trascendental. (A 138/B 177)

Los textos citados presentan diversas dificultades de interpretación. A continuación se mencionarán dos de ellas. En (2) se presenta un ejemplo de una relación de homogeneidad. Se afirma que el concepto empírico de plato guarda homogeneidad con el concepto geométrico de círculo en la medida en que la redondez, que es pensada en el primero, se puede intuir en el segundo. Los términos de la relación de homogeneidad son un concepto empírico y un concepto geométrico. Ahora bien, la homogeneidad había sido introducida en (1) como un requisito para la subsunción de un objeto bajo un concepto. Concepto y objeto deben ser homogéneos a fin de que el segundo sea subsumido bajo el primero. No se entiende, entonces, por qué Kant se ocupa en (2) de una relación de homogeneidad entre dos conceptos.

En (4) se indica que el esquema trascendental debe estar en homogeneidad tanto con la categoría como con el fenómeno. Según lo afirmado en (1) y en (3), parecería que el problema del esquematismo trascendental consiste en establecer una relación de homogeneidad entre categorías y multiplicidad empírica a fin de que haya subsunción. Los términos de la relación de homogeneidad serían, pues, las categorías y la multiplicidad empírica. No obstante, en (4) se ocupa Kant de relaciones de homogeneidad entre, por un lado, la categoría y el esquema trascendental, y, por el otro, el esquema trascendental y los fenómenos. No queda claro, entonces, cuáles son los términos de la relación de homogeneidad que debe establecer el esquematismo trascendental. 
A continuación se abordarán las dificultades mencionadas por medio de un análisis de las soluciones que han propuesto algunos comentaristas. Comenzaré con el segundo pasaje citado. El ejemplo del plato, la redondez y el círculo ha recibido diversas interpretaciones en la bibliografía crítica. Prueba de las dificultades de comprensión que presenta es que Hans Vaihinger ha propuesto una enmienda del texto. Según Vaihinger, la última parte de la oración debería leerse de la siguiente manera: "pues la redondez, que está pensada en el último, se puede intuir en el primero". Además, entiende que la expresión "el concepto empírico de un plato" es equívoca y tiene manifiestamente el sentido de "la representación empírica de un plato cualquiera". Estos comentarios críticos descansan - explica Vaihinger- en la hipótesis de que, en el ejemplo del plato, la redondez y el círculo, está en juego la oposición entre el objeto empírico intuitivo y el concepto abstracto. ${ }^{9}$ Tal vez pueda afirmarse que la enmienda de Vaihinger supone una interpretación del pasaje mencionado como ejemplificación de una relación de subsunción. En efecto, la afirmación de que en el pasaje en cuestión se opone el concepto abstracto al objeto intuitivo parece presuponer la hipótesis de que el ejemplo debe explicar cómo tiene lugar la subsunción del segundo bajo el primero.

La tesis de que (2) ejemplifica una relación de subsunción ha sido criticada por Mario Caimi en su trabajo sobre el concepto de homogeneidad en el capítulo del esquematismo. Caimi encuentra que la mayoría de los comentaristas operan con este supuesto, mas no coinciden en lo que toca al sentido y el valor del ejemplo. No hay unanimidad con respecto a qué es lo que debe ser subsumido, ni en relación con aquello bajo lo cual la subsunción ha de tener lugar. De hecho, los comentaristas oscilan, ante todo, entre una interpretación del ejemplo como caso de subsunción de un concepto empírico bajo un concepto geométrico y una interpretación como caso de subsunción de un objeto empírico bajo un concepto geométrico. ${ }^{10}$ Caimi propone una interpretación radicalmente diferente. El ejemplo en modo alguno sería un caso de subsunción, sino que tendría únicamente el propósito de mostrar lo que debe entenderse por homogeneidad en el contexto del capítulo del esquematismo. ${ }^{11} \mathrm{Di}$ -

${ }^{9}$ Cfr. H. Vaihinger, "Siebzig textkritische Randglossen zur Analytik", pp. 457458. La traducción es mía.

${ }^{10}$ Cfr. M. Caimi, "Der Teller, die Rundung, das Schema. Kant über den Begriff der Gleichartigkeit”, pp. 212-216.

${ }^{11}$ También Ernst Robert Curtius considera que el ejemplo no puede ilustrar una relación de subsunción; en cambio, estima que no es posible enlazar los conceptos de plato y círculo en un juicio de subsunción. En efecto, no se puede afirmar "el 
cho en otras palabras, el pasaje citado se limitaría a poner en evidencia que entre los conceptos de plato y círculo se establece una relación de homogeneidad en virtud de la introducción de un tercer elemento, es decir, la redondez. El tercer elemento tiene algo en común con los otros dos (a saber, el plato y el círculo) y posibilita así una vinculación entre el uno y el otro. El ejemplo apuntaría, entonces, a la explicación de un nuevo concepto de homogeneidad, el cual no guarda relación con la comprensión habitual de la palabra.

Nótese que los términos de la comparación son un concepto geométrico y un concepto empírico. No se trataría, entonces, de un caso de subsunción, pues entre dos conceptos se puede presentar más bien una relación de subordinación lógica. ${ }^{12}$ Son más bien los casos particulares los que pueden subsumirse bajo un concepto. Además, es preciso señalar que los términos comparados no son de la misma especie, pero ciertamente puede decirse que, en la medida en que ambos son conceptos, pertenecen al mismo género. Por tanto, los elementos son homogéneos en el sentido habitual del término. Ahora bien, Kant no considera que los conceptos sean homogéneos debido a que pertenecen al mismo género, sino en virtud de que la redondez, que es pensada en uno, se puede intuir en el otro. La apelación a un tercer elemento (a saber, la redondez) demuestra que el concepto de homogeneidad que está en cuestión no es el habitual. Si el objetivo del ejemplo fuese señalar que los conceptos son homogéneos en el sentido habitual de la expresión, no tendría sentido recurrir a la redondez como elemento común a ambos términos, dado que bastaría con el hecho de que éstos son conceptos. ${ }^{13}$

En (4) se afirma que el esquema trascendental es "un tercero". Esta caracterización ayuda a precisar el concepto de homogeneidad que ocupa nuestra atención. El esquema trascendental se comporta como la redondez de que habla el ejemplo del plato, la redondez y el círculo. En efecto, el esquema trascendental es un tercero que media entre dos elementos a fin de establecer una relación de homogeneidad entre ellos.

plato es un círculo". Ahora bien, Curtius considera que el ejemplo debería haber ilustrado la relación de subsunción. Curtius concluye, entonces, que el ejemplo es un "absoluto desacierto". Cfr. E.R. Curtius, "Das Schematismuskapitel in der Kritik der reinen Vernunft. Philologische Untersuchung", pp. 346-347; la traducción es mía. Es de señalar, también, que las tesis de Curtius las examina Caimi. Cfr. M. Caimi, op. cit., p. 213.

${ }^{12}$ Cfr. Lógica Jäsche, AA, IX, 95-100 (pp. 145-148 de la traducción en castellano).

${ }^{13}$ Cfr. M. Caimi, op. cit., pp. 216-217. 
Ciertamente, en el ejemplo mencionado no se trata de dos términos absolutamente heterogéneos como lo son las categorías y la multiplicidad empírica. Esta circunstancia se debe posiblemente a que Kant ha pretendido únicamente ilustrar, mediante aquél, el mecanismo de la homogeneidad, consistente en la introducción de un tercero mediador. Se advierte la novedad del concepto kantiano de homogeneidad: los elementos —en última instancia, entendimiento y sensibilidad- permanecen heterogéneos, en el sentido usual del término, incluso tras la mediación efectuada por el esquema trascendental. ${ }^{14}$ Ciertamente, no es posible que se establezca un vínculo de semejanza - u homogeneidad en sentido habitual - entre entendimiento y sensibilidad, pues ello implicaría el colapso de la distinción fundamental de la doctrina kantiana de las facultades cognitivas. La categoría y la multiplicidad empírica no entran en relación inmediatamente, sino por medio de un tercer término que tiene algo en común con ellas.

Considero que el artículo de Caimi presenta una interpretación satisfactoria del controvertido ejemplo del plato, la redondez y el círculo; además, caracteriza con precisión el concepto kantiano de homogeneidad. En lo que resta del presente artículo procuraré determinar aún más este concepto mediante la consideración de acepciones que se desprenden de otros pasajes y el examen crítico de la interpretación de Dieter Lohmar.

En su examen de (4), Lohmar identifica dos sentidos del concepto de homogeneidad. El primero, que indica homogeneidad del esquema con la categoría (homogeneidad $2 A$ ), consistiría en provenir únicamente del entendimiento, esto es, en ser intelectual. El segundo (homoge$\operatorname{neidad}_{2 B}$ ), que expresa homogeneidad del esquema con la intuición, radicaría en provenir solamente de la sensibilidad. ${ }^{15}$ La posición de Lohmar es que los esquemas trascendentales, entendidos - tal como se los definirá más adelante- como determinaciones trascendentales del tiempo (cfr. A 138/B 177), mantienen una relación de homogeneidad $2 A$ con las categorías. Por otra parte, agrega Lohmar, los esquemas trascendentales no guardan una relación de homogeneidad ${ }_{2 B}$ con las intuiciones, puesto que las determinaciones trascendentales del tiempo no se originan en la sensibilidad. Los esquemas trascendentales y las intuiciones, en cambio, sostendrían un vínculo de homogeneidad únicamente

${ }^{14}$ Cfr. M. Caimi, op. cit., pp. 219-220.

${ }^{15}$ Cfr. D. Lohmar: "Kants Schemata als Anwendungsbedingungen von Kategorien auf Anschauungen. Zum Begriff der Gleichartigkeit im Schematismuskapitel der Kritik der reinen Vernunft", p. 81. 
en la medida en que determinaciones del tiempo están contenidas en ambos. $^{16}$

Pienso que el afán de precisión exhibido por Lohmar en la determinación de los diferentes sentidos del concepto de homogeneidad es iluminador en lo que toca a los diversos matices que adquiere esta noción en distintos pasajes del capítulo del esquematismo. Con todo, considero que el concepto de homogeneidad decisivo para la doctrina del esquematismo es el que he expuesto antes con ayuda del ejemplo del plato, la redondez y el círculo. Ciertamente, al caracterizar el esquema trascendental como un tercero mediador, Kant habla de homogeneidad entre, por un lado, el esquema trascendental y la categoría, y, por el otro, el esquema trascendental y los fenómenos. Sin embargo, está claro que, en este contexto, tiene la preeminencia una comprensión habitual de la homogeneidad. La categoría y la multiplicidad empírica sostendrían, cada una por su parte, una relación de semejanza u homogeneidad (en sentido habitual) con el esquema trascendental. Ahora bien, el esquematismo trascendental procura producir homogeneidad entre las categorías y la multiplicidad empírica a fin de que haya subsunción. Por lo tanto, la relación de homogeneidad que está en cuestión es la que debe establecerse entre la categoría y la multiplicidad empírica. Tal relación de homogeneidad se consigue mediante la introducción del esquema trascendental, y no implica homogeneidad (en sentido habitual) entre los extremos de la relación. Por cierto, puede afirmarse, según lo establecido en (4), que esta peculiar relación de homogeneidad entre categoría y multiplicidad empírica supone relaciones de homogeneidad (en sentido habitual) entre, por un lado, la categoría y el esquema trascendental y, por el otro, el esquema trascendental y la multiplicidad empírica. ${ }^{17}$ Por consiguiente, si bien los conceptos de homogeneidad $2 \mathrm{~A}$ y homogeneidad ${ }_{2 B}$ distinguidos por Lohmar proporcionan una interpre-

${ }^{16}$ Cfr. D. Lohmar, op. cit., p. 82.

${ }^{17}$ Lauchlan Chipman considera que Kant interpreta la homogeneidad como no transitiva: las categorías y la multiplicidad empírica serían en cada caso homogéneas con el esquema trascendental, mas no entre sí (cfr. L. Chipman, "Kant's Categories and their Schematism", p. 42). Considero, en cambio, que la cuestión debe plantearse de la siguiente manera: entre categorías y multiplicidad empírica se establece una relación de homogeneidad —en el sentido kantiano del términoen virtud de la introducción de un tercer término con el cual cada una de ellas es homogénea —en el sentido habitual del término- - Nótese que Caimi, en el trabajo mencionado, no caracteriza como una relación de homogeneidad en sentido habitual el tipo de relación que sostienen, cada una por su parte, la categoría y la multiplicidad empírica con el esquema trascendental; en cambio, se ocupa exclusivamente de la relación de homogeneidad entre las categorías y las intuiciones. 
tación de relaciones de homogeneidad (en sentido habitual) supuestas por la relación de homogeneidad (en sentido kantiano), no permiten caracterizar adecuadamente esta última relación. ${ }^{18}$

Por otra parte, Lohmar considera que en (1) se efectúa una comparación entre concepto e intuición en términos de contenido. Su exégesis supone tener en consideración la distinción efectuada por Kant entre notas intuitivas y notas discursivas. Una nota intuitiva es una parte $-\mathrm{O}$ representación parcial- de la intuición en la medida en que puede concebírsela como un elemento constitutivo de lo intuido. Por ejemplo, en la intuición que tiene el lector de esta página está incluida, como contenido intuitivo, la propiedad "rectangular". "Rectangular" ha de entenderse aquí como este diseño rectangular concreto, provisto de un color y un tamaño determinados. Los conceptos "rectángulo" y "cuerpo", en cambio, son ejemplos de notas discursivas y pueden ser considerados como partes integrantes del concepto "hoja de papel"; es decir, constituyen representaciones universales de las cosas, bajo las cuales pueden subsumirse uno o más cuerpos rectangulares. Puede afirmarse, entonces, que el contenido de un concepto viene dado por las notas discursivas que lo integran. Análogamente, el contenido de una intuición consistiría en el conjunto de las notas intuitivas dadas en ella. Nótese, además, que una nota discursiva posee el mismo contenido objetivo que la nota intuitiva correspondiente. La diferencia de carácter entre ambas tiene su origen en la facultad de conocimiento (entendimiento o sensibilidad) con la que en cada caso se vinculan. ${ }^{19}$

\footnotetext{
${ }^{18}$ Hay que señalar que los conceptos de homogeneidad ${ }_{2 A}$ y homogeneidad hom $_{2 B}$ distinguidos por Lohmar, así como la crítica que efectúa de homogeneidad ${ }_{2 B}$, suponen una interpretación del concepto de determinación trascendental del tiempo. En efecto, el esclarecimiento de las relaciones de homogeneidad (en sentido habitual) entre, por un lado, la categoría y el esquema trascendental y, por el otro, el esquema trascendental y la multiplicidad empírica, presuponen una comprensión acabada del concepto de esquema trascendental. Ahora bien, el análisis exhaustivo de este concepto excede los límites del presente trabajo. Aquí me limitaré a indicar que los conceptos de homogeneidad ${ }_{2 A}$ y homogeneidad ${ }_{2 B}$ no proporcionan una interpretación adecuada del novedoso concepto de homogeneidad acuñado por Kant en el capítulo del esquematismo. Más adelante se ofrecerán indicaciones mínimas acerca del concepto de esquema trascendental y de cómo podrían concebirse las relaciones entre, por un lado, la categoría y el esquema trascendental y, por el otro, el esquema trascendental y la multiplicidad empírica.

${ }^{19}$ Kant expone el concepto de nota común o discursiva en Lógica Jäsche. Cfr. AA, IX, 58-65, 95 (pp. 118-124, 145 de la traducción española). El concepto de nota intuitiva es introducido en la Reflexión no. 2286 (AA, XVI, 299-300; cfr. también la Reflexión no. 2282, AA, XVI, 298). Me he servido del análisis del concepto de nota
} 
En (1) se establece que, a fin de que un objeto sea subsumido bajo un concepto, éste debe ser homogéneo con aquél. A renglón seguido se explica esta homogeneidad de la siguiente manera: el concepto debe contener aquello que está representado en el objeto. Si tenemos en cuenta el análisis precedente de los conceptos de nota discursiva y nota intuitiva, podemos comprender las declaraciones de (1) en el sentido de que el concepto debe contener las notas discursivas correspondientes a las notas intuitivas dadas en una intuición. Lohmar considera que debe interpretarse "aquello" (dasjenige) — cuyo significado Kant no aclara en el texto- como una expresión que hace referencia a que todas las notas discursivas del concepto tienen que estar presentes en la intuición. En otras palabras, no podría haber nota discursiva del concepto que no esté dada como nota intuitiva en la intuición. ${ }^{20}$ Lohmar estima que la acepción examinada constituye un requisito fuerte de homogeneidad y la denomina homogeneidad ${ }_{1 A} \cdot{ }^{21}$

Además, Lohmar considera que Kant presenta en (2) una interpretación diferente del concepto de homogeneidad. Esta nueva perspectiva $\left(\right.$ homogeneidad $_{1 B}$ ) supondría una comprensión más débil del requisito de homogeneidad. En efecto, los dos conceptos mencionados — "plato" y "círculo" - son calificados de homogéneos, aparentemente, en virtud de la posesión de una única nota en común, a saber, la redondez. La extensión de este punto de vista a la problemática de la subsunción tiene como consecuencia, supone Lohmar, que la intuición sea susceptible de ser subsumida bajo un concepto únicamente cuando al menos una nota intuitiva incluida en la primera es pensada en el segundo. ${ }^{22}$ Considero que esta interpretación de Lohmar es incorrecta. En efecto, mis análisis han establecido que, con el ejemplo del plato, la redondez y el círculo, se indica que la categoría y la multiplicidad empírica deben ser puestas en relación mediante un tercer elemento. El tercer elemento se muestra más adelante como el esquema trascendental. No se trata, entonces, de que el concepto y la intuición deban poseer al menos una nota en común para que la subsunción tenga lugar.

intuitiva efectuado por Houston Smit; de este último, cfr. "Kant on Marks and the Immediacy of Intuition".

${ }^{20} \mathrm{Cfr}$. D. Lohmar, op. cit., p. 80. Está de más decir que no es posible que el requisito de homogeneidad consista en que el concepto debe contener todas las notas dadas en la intuición. Los conceptos son representaciones parciales y en modo alguno pueden albergar la totalidad de las notas ofrecidas por la intuición. Cfr. Lógica Jäsche, AA, IX, 99 (p. 147 de la versión en castellano).

${ }^{21}$ Cfr. D. Lohmar, op. cit., p. 80.

${ }^{22}$ Cfr. D. Lohmar, op. cit., pp. 80-81. 
Ahora bien, aún no se ha evaluado aquí la pertinencia del concepto de homogeneidad ${ }_{1 A}$ establecido por Lohmar ni su tesis de que en el capítulo del esquematismo se comparan la categoría y la intuición en términos de contenido. En (3) se afirma que las categorías nunca pueden ser halladas en intuición alguna. Esta afirmación pone en evidencia la comparación con respecto al contenido señalada por Lohmar. Las categorías, como todos los conceptos, provienen del entendimiento en lo que respecta a su forma, pero - a diferencia de los conceptos empíricos- también en lo que toca a su contenido. ${ }^{23}$ Por lo tanto, tal contenido es heterogéneo con respecto a las notas intuitivas dadas a la sensibilidad. En síntesis, al contenido de las categorías no le corresponden notas intuitivas. La heterogeneidad entre categorías y multiplicidad empírica se deriva, entonces, de la heterogeneidad que se presenta entre el entendimiento y la sensibilidad. Sirviéndonos de la terminología de Lohmar, podemos aseverar que los conceptos empíricos, en cambio, mantienen una relación de homogeneidad ${ }_{1 A}$ con la multiplicidad empírica. Dicho en otras palabras, la totalidad de las notas que los componen puede ser hallada en la intuición empírica. ${ }^{24}$

Considero que es acertada la tesis de Lohmar acerca de que en el primer párrafo del capítulo del esquematismo está en juego una comparación entre categorías y multiplicidad empírica en términos de contenido. Asimismo, pienso que lo que Lohmar llama homogeneidad ${ }_{1 A}$ (a saber, el requisito de que la intuición debe presentar todas las notas concebidas en el concepto) tiene cierta incidencia en la problemática del esquematismo trascendental. Ahora bien, ¿cómo es posible que el contenido de las categorías de alguna manera sea hallado en las intuiciones empíricas, siendo así que Kant lo niega expresamente? Esta dificultad se resuelve señalando que, ciertamente, la multiplicidad empírica por sí sola no exhibe el contenido propio de las categorías, aunque sí presentan algo análogo a él los objetos empíricos constituidos como tales. No es posible, entonces, que se establezca una relación de homogeneidad $_{1 A}$ entre categorías y multiplicidad empírica. ${ }^{25}$ Con todo,

${ }^{23}$ Cfr. Lógica Jäsche, AA, IX, 92 (p. 142 de la versión en castellano).

${ }^{24}$ Podría objetarse que Kant también defiende la necesidad de un esquematismo de los conceptos empíricos, e incluso de los matemáticos (cfr. A 140-142/B 179181). Ahora bien, en este caso, el problema al que se aboca el esquematismo no consiste en una heterogeneidad entre el contenido del concepto y el de la intuición, sino en una diferencia entre la universalidad del concepto y la singularidad de lo dado en la intuición. Para un examen del problema, cfr. J.P. Nolan, "Kant on Meaning: Two Studies", pp. 122-123.

${ }^{25}$ En este punto estamos de acuerdo con Lohmar. Cfr. D. Lohmar, op. cit., p. 81. 
la producción de homogeneidad — tal como la concibo-, es decir, la mediación del esquematismo trascendental, permite, según Kant, que la subsunción tenga lugar y que se establezca así un vínculo análogo a homogeneidad $\mathrm{d}_{1 A}$ entre las categorías y los objetos empíricos que son constituidos mediante la subsunción.

La "Analítica de los principios", precisamente, tiene por cometido que un análogo ${ }^{26}$ temporal del contenido de cada categoría sea impuesto a la multiplicidad empírica, a fin de que ésta sea constituida como objeto empírico. Este análogo temporal no es sino el esquema trascendental, elemento mediador entre categoría y fenómenos. Mediante el esquema trascendental se establece una peculiar relación de homogeneidad entre los términos mencionados. Tales términos permanecen heterogéneos en el sentido usual de la palabra.

Tal vez sea conveniente aclarar la cuestión mediante un ejemplo. En la categoría de sustancia y accidente se piensa la unidad de la síntesis entre un sujeto (que no puede ser predicado de otra cosa) y un predicado. Tal síntesis se dirige a una multiplicidad en general. Hasta aquí no nos encontramos con determinación temporal alguna. En efecto, no sabemos qué determinaciones debe poseer el fenómeno que, sin ser predicado, oficie de sujeto en la experiencia. Dicho en otras palabras, no sabemos qué determinaciones empíricas debe poseer una sustancia. En síntesis, la categoría pura de sustancia y accidente posee una significación meramente lógica. ${ }^{27}$ No hay, entonces, referencia al tiempo en la categoría mencionada. Ahora bien, el análogo temporal de la categoría de la sustancia y accidente es su esquema. Tal esquema es producido por la imaginación en un esfuerzo por interpretar el contenido de la categoría en términos de la experiencia posible. ${ }^{28}$ Kant caracteriza el

${ }^{26}$ Para un análisis de este sentido del término "analogía”, cfr. H.J. Paton, Kant's Metaphysic of Experience, t. II, pp. 180-183, y H.E. Allison, El idealismo trascendental de Kant: una interpretación y defensa, pp. 304-307.

${ }^{27}$ Cfr. B 150-152, A 147/B 186-187.

${ }^{28}$ Podría objetarse que el esquema no consiste en la interpretación del contenido de la categoría en términos de la experiencia posible, sino en términos de la intuición pura del tiempo. En efecto, Kant define el esquema trascendental como "transzendentale Zeitbestimmung" (A 138/B 177). Esta expresión es traducida habitualmente como "determinación trascendental del tiempo". Ahora bien, la expresión mencionada también puede ser traducida como "determinación trascendental temporal". Según esta interpretación, el esquema no sería una determinación del tiempo puro, sino una determinación temporal de intuiciones empíricas posibles. La caracterización del esquema trascendental de la categoría de sustancia y accidente, a mi juicio, confirma la segunda interpretación. En efecto, tal esquema trascendental consiste en la relación temporal (permanencia/mutabilidad) entre dos factores 
esquema mencionado de la siguiente manera: "la permanencia de lo real en el tiempo, es decir, la representación de ello como un substrato de la determinación empírica general del tiempo, [substrato] que permanece, pues, mientras todo lo demás cambia". ${ }^{29}$ Considero que el pasaje citado puede interpretarse en el sentido de que el esquema trascendental de la categoría de sustancia y accidente consiste en la relación entre algo real permanente (la sustancia) y algo real mudable (el accidente). ${ }^{30}$

La relación entre algo real permanente y algo real mudable (el esquema trascendental) es el análogo temporal de la relación entre un sujeto (que no puede ser predicado de otra cosa) y un predicado (la categoría pura). Mediante la introducción de este tercer elemento (a saber, el esquema de la categoría de sustancia y accidente) se produce la homogeneidad entre multiplicidad empírica y categoría que es requisito para la subsunción.

Por último, el esquema es establecido como característica fundamental de la objetividad en la primera de las "Analogías de la experiencia". En este punto, la subsunción ya ha tenido lugar. Un procedimiento universal de síntesis, ejecutado por la imaginación y regido por la categoría de sustancia y accidente, ha unificado la multiplicidad empírica en general de modo que quede estructurada como sustancia(s) permanente(s) dotada(s) de accidentes mudables. ${ }^{31}$ La interpretación temporal de la categoría ha sido impuesta a la multiplicidad empírica,

fenoménicos. Cfr. infra. Tanto Paton como Allison y Düsing han afirmado que el esquema trascendental no consiste en una determinación del tiempo puro, sino en una determinación temporal de lo empírico. Cfr. H.J. Paton, Kant's Metaphysic of Experience, t. II, pp. 28-30; H.E. Allison, El idealismo trascendental de Kant: una interpretación y defensa, pp. 286-287; y K. Düsing, "Schema und Einbildungskraft in Kants Kritik der reinen Vernunft", p. 69. Por último, debo señalar que he conservado la traducción de "transzendentale Zeitbestimmung" como "determinación trascendental del tiempo" en virtud de que es la más habitual. M. Caimi, en la p. 244, n. 525, de su edición de la Crítica de la razón pura, da noticia de la traducción alternativa que prefiero.

${ }^{29}$ A $144 /$ B 183.

${ }^{30}$ Coincido en este punto con Longuenesse. Cfr. B. Longuenesse, Kant and the Capacity to Judge, p. 332.

${ }^{31}$ La tarea de determinar si Kant sostiene una concepción monista o pluralista de la sustancia supone un análisis de textos que no es pertinente llevar a cabo aquí. Puede consultarse con provecho la discusión del tema ofrecido por Allison, $E l$ idealismo trascendental de Kant: una interpretación y defensa, pp. 321-333. Por su parte, Rosales ha presentado una reconstrucción de los tipos de acciones de síntesis de la imaginación que dan origen a una sustancia permanente dotada de accidentes mudables. Cfr. A. Rosales, "Una pregunta sobre el tiempo", pp. 236-239. 
con lo cual es objetivada. La definición del principio en cuestión según la edición de 1781 es la siguiente: “Todos los fenómenos contienen lo permanente (sustancia) como el objeto mismo, y lo mudable, como mera determinación de aquél, es decir, [como] un modo como el objeto existe" (A 182).

El objeto empírico constituido como tal presenta una característica temporal (la relación entre la sustancia y el accidente fenoménicos) análoga al contenido de la categoría (la relación entre un sujeto último $\mathrm{y}$ un predicado en tanto que unifica una multiplicidad en general). ${ }^{32}$ Las categorías y los objetos empíricos sostienen, entonces, una relación análoga a la homogeneidad ${ }_{1 A}$ de Lohmar. Con todo, esta relación no constituye el sentido del concepto de homogeneidad más relevante para la doctrina del esquematismo. Nuestro análisis ha mostrado que la relación mencionada no es un requisito para la subsunción, sino una consecuencia de la misma. En efecto, la multiplicidad empírica es subsumida bajo las categorías y, por consiguiente, es constituida como objeto empírico en el momento mismo en que, en virtud de la síntesis de la imaginación, presenta la relación en cuestión. Por lo tanto, la relación mencionada es una propiedad de los objetos empíricos constituidos como tales. Ahora bien, la homogeneidad es introducida en el

${ }^{32}$ Este ejemplo presupone una interpretación de los esquemas trascendentales como propiedades o características de los objetos empíricos. No se intenta afirmar que esta definición proporcione una comprensión acabada de la naturaleza de los esquemas trascendentales; sin embargo, la definición en cuestión es válida como primera aproximación y, por lo tanto, útil a los fines de un ejemplo. Señalemos que esta interpretación fue sostenida por Paton, Detel y Düsing. Cfr. H.J. Paton, Kant's Metaphysic of Experience, t. II, pp. 28-30, 43; W. Detel, "Zur Funktion des Schematismuskapitels in Kants Kritik der reinen Vernunft", pp. 42-43 (sin embargo, cfr. pp. 40-41) y K. Düsing, "Schema und Einbildungskraft in Kants Kritik der reinen Vernunft", p. 69. Por último, nótese que también es posible afirmar que los esquemas trascendentales no son tanto propiedades temporales de los objetos empíricos, sino más bien los procedimientos universales de síntesis que unifican la multiplicidad empírica de modo de producir las propiedades mencionadas. En efecto, en Crítica de la razón práctica, Kant se refiere al esquema trascendental como a un "procedimiento universal de la imaginación" (AA, V, 65). Esta ambigüedad en la concepción kantiana de los esquemas trascendentales no constituye una incoherencia, dado que las concepciones mencionadas no son incompatibles. Tal vez pueda afirmarse que los esquemas trascendentales son tanto procedimientos de síntesis de la imaginación cuanto las propiedades temporales que resultan de tales procedimientos. Me limitaré a señalar la plausibilidad de esta interpretación, cuya presentación exigiría una argumentación exhaustiva. A los fines del ejemplo que aquí se trata, basta con determinar los esquemas trascendentales como propiedades temporales de los objetos empíricos. 
capítulo del esquematismo como un requisito para la subsunción de la multiplicidad empírica bajo las categorías, y consiste, por lo tanto, en un vínculo que debe establecerse entre los términos mencionados.

Resumamos las conclusiones a las que hemos llegado. Los esquemas trascendentales son elementos mediadores entre las categorías y la multiplicidad empírica en la medida en que constituyen un tercer elemento que tiene algo en común con ambos términos. La relación de homogeneidad entre categorías y multiplicidad empírica se establece en virtud de la mediación de los esquemas trascendentales. Además, los esquemas trascendentales sostienen una relación de homogeneidad (en el sentido habitual del término) tanto con las categorías como con la multiplicidad empírica. Los esquemas son semejantes a las categorías, pues constituyen análogos temporales ellas. Son también semejantes a los fenómenos, dado que consisten en análogos temporales de las categorías, y todos los fenómenos nos son dados bajo la forma del tiempo. ${ }^{33}$ Ahora bien, la introducción del esquema trascendental no supone una supresión de la heterogeneidad (en el sentido usual de la palabra) entre los términos que vincula. En efecto, las categorías, consideradas haciendo abstracción de sus esquemas, se refieren meramente a una multiplicidad en general y no tienen vínculo alguno con la intuición humana. La homogeneidad kantiana consiste, entonces, en una relación entre dos términos heterogéneos conseguida mediante la introducción de un tercer elemento que tiene algo en común con ambos términos.

\section{BIBLIOGRAFÍA}

\section{1) Fuentes y traducciones}

Kant, I., Kant's gesammelte Schriften, edición de la Königlich Preußischen Akademie der Wissenschaften, Berlín, 1902 ss.

—- Crítica de la razón pura, trad. Mario Caimi, Colihue, Buenos Aires, 2007. —_, Lógica, trad. María Jesús Vázquez Lobeiras, Akal, Madrid, 2000.

${ }^{33}$ Debo señalar que Dieter Lohmar considera que las relaciones de homogeneidad entre, por un lado, la categoría y el esquema trascendental y, por el otro, el esquema trascendental y la multiplicidad empírica son diferentes (cfr. supra). La mediación del esquema trascendental se basaría entonces, según Lohmar, en un empleo oscuro de dos conceptos de homogeneidad diferentes, aunque designados por el mismo nombre. Cfr. D. Lohmar, op. cit., p. 82. No es posible examinar aquí esta objeción; para los fines de este artículo, basta con caracterizar las relaciones mencionadas como relaciones de semejanza u homogeneidad en sentido habitual. No se ha pretendido evaluar el éxito de la mediación efectuada por el esquema trascendental, sino simplemente esclarecer el sentido del concepto kantiano de homogeneidad. (Cfr. n. 18, supra). 
2) Obras de referencia

Der Digitale Grimm. Deutsches Wörterbuch von Jacob und Wilhelm Grimm, edición digital en dos discos compactos, ed. Hans-Werner Bartz, Thomas Burch, Ruth Christmann, Kurt Gartner, Vera Hildebrandt, Thomas Schares y Klaudia Wegge, Zeitausendeins, Fráncfort del Meno, 2004.

Wahrig Deutsches Wörterbuch (edición digital), Bertelsmann Lexikon Verlag/ Bertelsmann Electronic Publishing, Gütersloh/Munich, 1997.

\section{3) Bibliografía crítica}

Allison, H.E., El idealismo trascendental de Kant: una interpretación y defensa, trad. Dulce María Granja Castro, Anthropos, Barcelona, 1992; versión en castellano de: Kant's Transcendental Idealism: An Interpretation and Defense, Yale University Press, New Haven/New York, 1983.

Caimi, M., "Der Teller, die Rundung, das Schema. Kant über den Begriff der Gleichartigkeit", en Dirk Fontana (comp.), Metaphysik als Wissenschaft. Festschrift für Klaus Düsing zum 65. Geburtstag, Alber, Friburgo/Munich, 2006, pp. 211-220.

Chipman, L., "Kant's Categories and their Schematism", Kant Studien, vol. 63, no. 1, 1972, pp. 36-50.

Curtius, Ernst Robert, "Das Schematismuskapitel in der Kritik der reinen Vernunft. Philologische Untersuchung", Kant Studien, vol. 19, 1914, pp. 338366.

Daval, R., La Métaphysique de Kant. Perspectives sur la métaphysique de Kant d'après la théorie du schématisme, Presses Universitaires de France, París, 1951.

Detel, W., "Zur Funktion des Schematismuskapitels in Kants Kritik der reinen Vernunft", Kant Studien, vol. 69, no. 1, 1978, pp. 17-45.

Düsing, K., "Schema und Einbildungskraft in Kants Kritik der reinen Vernunft", en Lothar Kreimendahl (comp.), Aufklärung und Skepsis. Studien zur Philosophie und Geistesgeschichte des 17. und 18. Jahrhunderts. Günter Gawlick zum 65, Geburtstag, Frommann-Holzboog, Stuttgart-Bad Cannstatt, 1995, pp. 47-71.

Ferrarin, A., "Construction and Mathematical Schematism. Kant on the Exhibition of a Concept in Intuition", Kant Studien, vol. 86, no. 2, 1995, pp. 131174.

Lohmar, D., "Kants Schemata als Anwendungsbedingungen von Kategorien auf Anschauungen. Zum Begriff der Gleichartigkeit im Schematismuskapitel der Kritik der reinen Vernunft", Zeitschrift für philosophische Forschung, vol. 45, no. 1, 1991, pp. 77-92.

Longuenesse, B., Kant and the Capacity to Judge. Sensibility and Discursivity in the Transcendental Analytic of the Critique of Pure Reason, trad. Charles T. Wolfe, Princeton University Press, Princeton, 2000; versión en inglés de 
Kant et le pouvoir de juger. Sensibilité et discursivité dans l'Analytique transcendantale de la Critique de la raison pure, Presses Universitaires de France, París, 1993.

Nolan, J.P., "Kant on Meaning: Two Studies", Kant Studien, vol. 70, no. 2, 1979, pp. 113-130.

Paton, H.J., Kant's Metaphysic of Experience. A Commentary on the First Half of the Kritik der reinen Vernunft, 5a. ed., George Allen and Unwin, Londres, 1970, 2 vols.

Pippin, R.B., "The Schematism and Empirical Concepts", Kant Studien, vol. 67, no. 2, 1976, pp. 156-171.

Rosales, A., "Una pregunta sobre el tiempo", en Siete ensayos sobre Kant, Universidad de los Andes, Mérida, 1993.

Smit, H., "Kant on Marks and the Immediacy of Intuition", The Philosophical Review, vol. 109, no. 2, 2000, pp. 235-266.

Vaihinger, H., "Siebzig textkritische Randglossen zur Analytik", Kant Studien, vol. 4, 1900, pp. 452-463.

Winterbourne, A.T., "Construction and the Role of Schematism in Kant's Philosophy of Mathematics", Studies in History and Philosophy of Science, vol. 12, no. 1, 1981, pp. 33-46.

Young, M., "Construction, Schematism, and Imagination", Topoi, vol. 3, no. 2, 1984, pp. 123-131.

Recibido el 5 agosto de 2008; aceptado el 5 de mayo de 2009.

Diánoia, vol. LIV, no. 63 (noviembre 2009). 